\title{
Long-term results after restoring nasal tip support using auricular cartilage as an intercrural columellar strut graft: the "I-Beam" technique*
}

\author{
Eva Novoa', Daniel Simmen², Hans Rudolf Briner ${ }^{2}$, Christoph Schlegel' \\ ' Department of Otorhinolaryngology, Head, Neck and Facial Plastic Surgery, Luzerner Kantonsspital, Lucerne, Switzerland \\ 2 ORL-Zentrum, Center for Rhinology, Skull Base Surgery and Facial Plastic Surgery, The Hirslanden Clinic, Zurich, Switzerland
}

Rhinology 56; 2: 183-188, 2018

https://doi.org/10.4193/Rhin17.184

*Received for publication:

August 15, 2017

Accepted: January 11, 2018

Background: The lack of nasal tip support resulting in poor protection and projection of the nose is a common cause of nasal obstruction and cosmetic impairment. Although septal cartilage is preferred for restoration of nasal tip support, there is often not enough septal cartilage available. We describe an intercrural columellar strut graft in a circumferential soft tissue pocket: the "I-Beam" technique.

Methodology: In this cohort study over an 8-year period the clinical and biometrical long-term results after restoration of nasal tip support with an autologous auricular cartilage graft, the "I-Beam" technique, is evaluated.

Results: Out of the 72 consecutive septorhinoplasties with the "I-Beam" technique, we studied 66 patients in the median longterm follow-up of 89 months. In all patients a significant improvement in nasal tip projection was found. A functional improvement in nasal breathing was achieved in $86 \%$. The overall satisfaction with the "I-Beam" graft was of $83 \%$. No deterioration in smell perception was found after surgery.

Conclusions: The "I-Beam" technique using back-to-back sutured autologous auricular cartilage is a safe and effective technique and gives long-term structural support and enhances tip projection.

Key words: rhinoplasty, ear cartilage, septal nasal cartilage, aesthetics, follow-up studies

\section{Introduction}

The lack of nasal tip support and the resulting poor protection and projection of the nose is a common cause of nasal obstruction and cosmetic impairment. Previous nasal surgeries with aggressive resection of the caudal septum, trauma, infection or congenital deformities are some of the main causes. According to the Tardy's classification, the caudal end of the quadrangular cartilage is a crucial intrinsic mechanism in nasal tip support ${ }^{(1)}$. Furthermore ptosis (loss of protection) of the nasal tip affects the internal nasal valve with deformation of the angle between the septum and upper lateral cartilages ${ }^{(2)}$. Therefore, restoring the caudal septum and tip support is important in improving function as well as the aesthetics of the nose. Restoration of the caudal septum and nasal tip support with a caudal septal ex- tension graft or a columellar strut graft with any residual septal cartilage is preferable when enough septal cartilage remains. However, after trauma or previous surgery there is often not enough septal cartilage available and alternative graft materials such as rib, conchal cartilage or bone have been used ${ }^{(3)}$.

\section{The "I-Beam" technique}

The "I-Beam" graft is a type of an intercrural columellar strut graft and uses back-to-back sutured autologous conchal cartilage. It was introduced by McCollough ${ }^{(4)}$ and Simmen ${ }^{(5)}$. The term "I-Beam" is derived from its morphologic resemblance with the I-shaped beams used in construction and civic engineering made of structural steel. Due to its structural strength it is a very powerful graft and reconstitutes protection of the tip without 


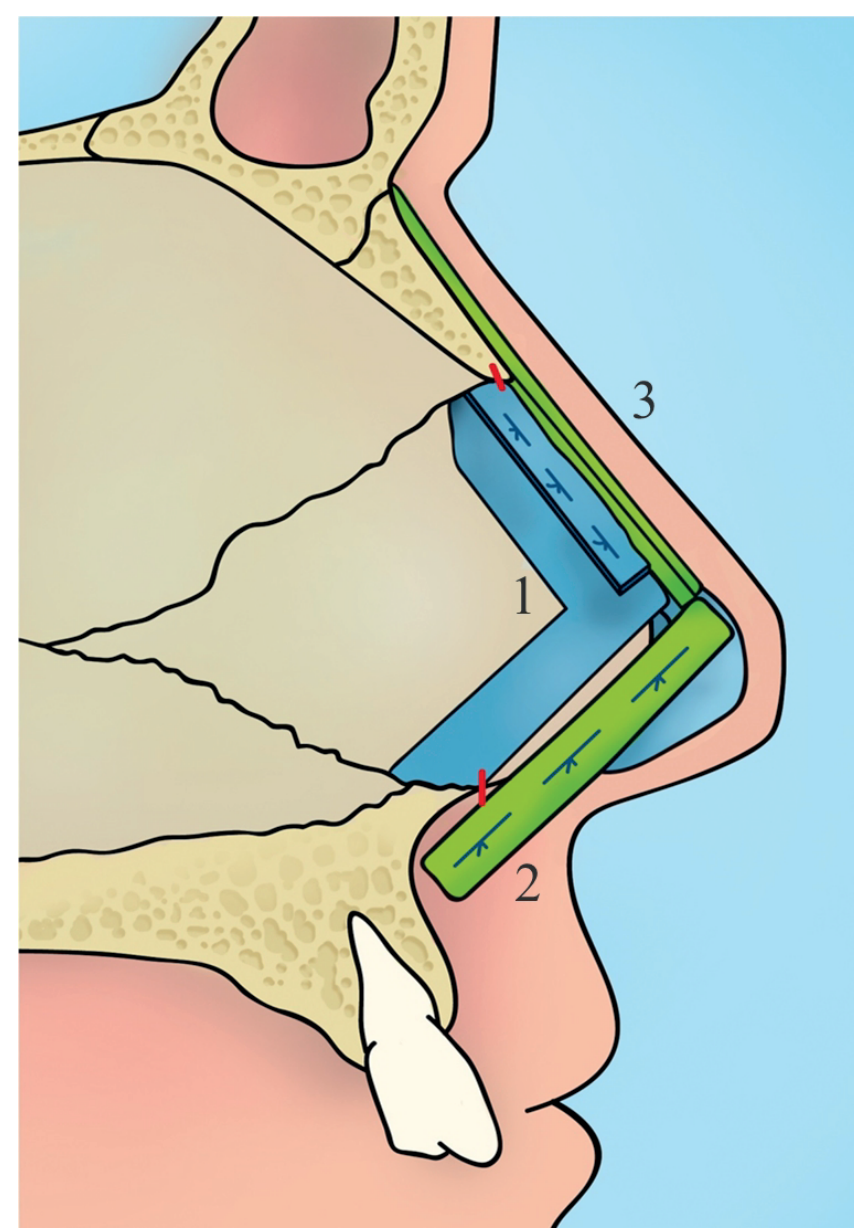

Figure 1. Diagram showing reconstruction of saddle nose deformity. Corrected remaining septum is straightened with spreader grafts (1). The "I-Beam" gives protection and projection of the tip and enhances columellar show (2). Dorsal onlay graft refills saddle deformity (3).

the rigidity of rib cartilage or bone. This graft maintains tip support as it restores the nasolabial angle (NLA) providing enough support, stability and projection. Contrary to a caudal septal extension graft, the "I-Beam" is not sutured to the remaining septum, but is placed more anterior in a circumferential soft tissue pocket in the columella. The goal of this study was to evaluate the long-term functional and cosmetic results of reconstituting the nasal tip support using the "I-Beam" back-to-back sutured autologous conchal cartilage graft technique.

\section{Materials and methods}

Over an eight-year time period all consecutive patients with a septorhinoplasty using the "I-Beam" technique to correct a significant loss in their nasal tip support (loss of projection, protection, retracted columella) were analysed as a cohort. All patients had a significant lack of cartilage in the caudal part of the septum (regio I). The study was evaluated and approved by the Ethics in Research Committee of our institution. Inclusion criteria were patients older than 16 years undergoing septorhinoplasty with an "I-Beam" along with pre- and postoperative digital images and a follow-up of more than 2 years. All procedures were carried out by the senior author.

\section{Surgical technique}

In case of relevant septal deviation, a septoplasty is carried out first of all through a classic hemitransfixion incision leaving the membranous septum intact. Weak or deeply distorted caudal septal cartilage with no more function for structural support is resected. The remaining septum is corrected by classic means like posterior chondrotomy or re-fixation in the midline with a figure-of-eight suture to the anterior nasal spine ore more posterior to the premaxilla. If there is a relevant inferior turbinate hypertrophy an endoscopic turbinoplasty with submucous resection of a hypertrophic turbinate bone and its insertion at the frontal process of maxilla is performed. We do not correct the septum through an open approach, because an intact circumferential columella pocket is essential for the "I-Beam" technique. After correcting the remaining septum, an external rhinoplasty approach is performed. If there was a need to correct the bony vault like in deviated noses or hump deformities, osteotomies or hump resection are done. Then a distorted middle vault is stabilised and straightened with spreader grafts or augmented in saddle noses, if necessary. At the end of the procedure, the missing caudal septum (regio I) is reconstructed and nasal tip support is restored inserting the "I-Beam" as an intercrural columellar strut graft (Figure 1). Based on the principle of the back-to-back autologous auricle cartilage graft proposed by Pirsig ${ }^{(6)}$ an entire cymba-cavum conchae complex is harvested. We prefer a retroauricular incision following the contour of the posterior conchal wall. This is extended anteriorly as much as possible to harvest as much of the cymba and conchal bowl, including the less regular part that almost starts to form the root of the helix. Generally, the length obtained from the conchal cartilage graft is more than $30 \mathrm{~mm}$, which allows to insert a 24-26 $\mathrm{mm}$ long "I-Beam" tailored to the needs of patient's anatomy. The remaining auricular cartilage can be used for other reconstructive manoeuvres. By incising in the trimmed concave (anterior) part in a longitudinal way and leaving the perichondrium of the convex (posterior) part intact, the conchal cartilage is divided and folded on itself. The two strips of curved cartilage are joined with several 5-0 nylon mattress sutures so that a straight strut results (Figure 2). To avoid an auricular hematoma transfixion mattress sutures are placed through a small piece of gauze that is sutured on both sides of the conchae.

Through an open rhinoplasty approach a subcutaneous pocket in the columella between the medial crura down toward the anterior nasal spine is made being careful to ensure that the membranous septum is kept intact. A good-sized pocket is required to take the graft down to, and just in front of, the anterior 

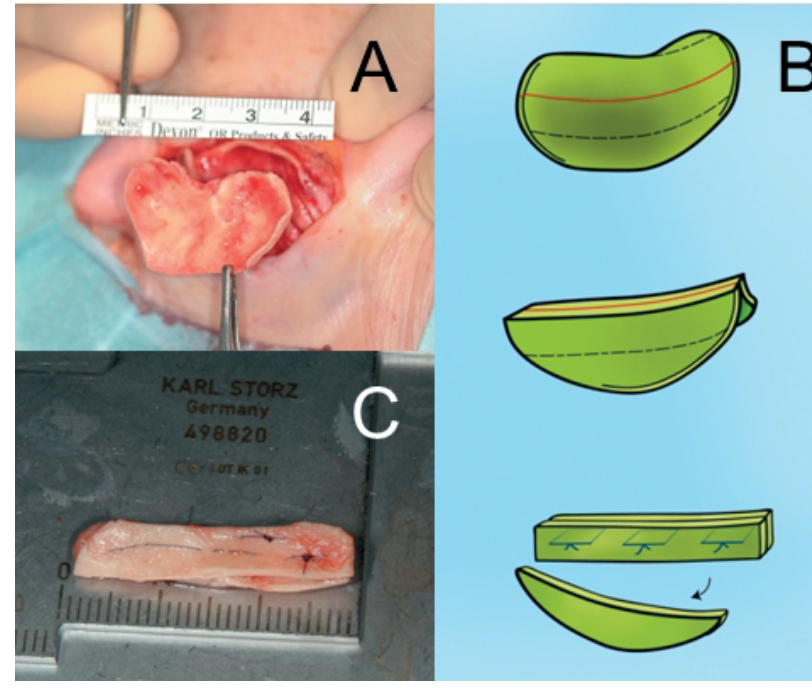

Figure 2. A) An entire cymba-cavum conchae complex is harvested by a retroauricular incision. B) By incising in the trimmed concave part in a longitudinal way and leaving the perichondrium of the convex part intact, the conchal cartilage is divided and folded on itself. C) The two strips of curved cartilage are joined with mattress sutures so that a straight strut results.

nasal spine. A guiding suture (Prolene 3-0) is then placed from the base of the columella through the pocket into the lower end of the "I-Beam" and back again to pull the graft optimal at the very deep end of the pocket (Figure 3). Transfixion sutures secure the "I-Beam" safely between the medial crura of the lower lateral cartilages and in the circumferential soft tissue pocket. If needed transdomal and interdomal sutures are added (Figure 4). Consequently, the base of the graft is not sutured allowing some curling similar to the feet of the medial crura on and in front of the nasal spine.

\section{Long-term follow up patient evaluation}

The surgical records of all rhinoplasties performed by the senior author were reviewed and those patients who qualified for the study were contacted by phone or letter and were invited for a follow-up examination in our outpatient clinic. An informed consent was provided.

Focus in long-term follow up was on objective measurements of nasal tip projection using computerized biometrical analysis comparing pre- and postoperative images. Further evaluation comprised clinical examination and, off target, subjective assessment by VAS. Biometrical analysis of nasal tip projection was performed based on the iris-dependent calibration technique described by Spoerri et al. ${ }^{(5)}$. By applying this method, it is possible to compare not only ratios to evaluate nasal tip projection (7), but also distances and facial angles in pictures taken at different moments during the follow-up based on a constant size of the iris diameter in adults of $11 \pm 0.4 \mathrm{~mm}$. To measure nasal tip

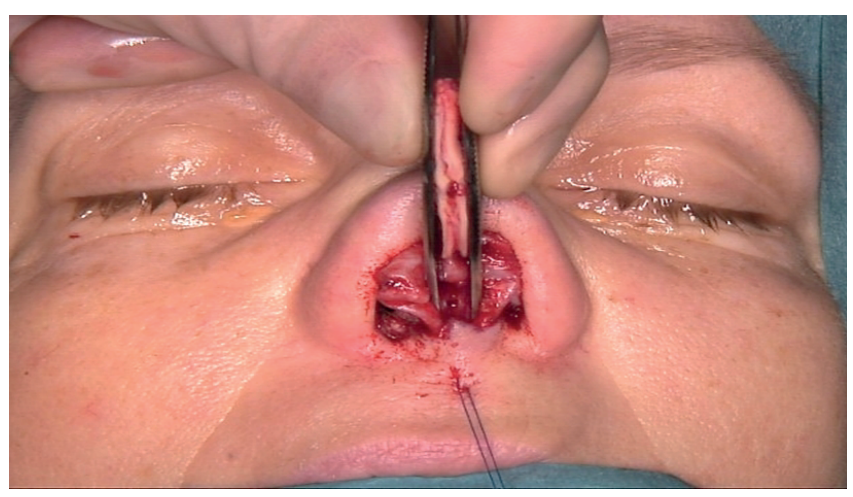

Figure 3. The "I-Beam" is placed with a guiding suture into the circumferential columellar soft tissue pocket.

projection the method proposed by Crumley and Lanser ${ }^{(8)}$ was used. A line from the vertex of the naso-frontal angle (nasion) to the vermilion-cutaneous junction of the upper lip is drawn. The perpendicular to this line to the tip defining point is measured as nasal tip projection. Computerized measurements and comparisons between pre- and postoperative digital images were performed with the support of the freeware MB-Ruler ${ }^{\oplus}$. The postoperative clinical examination included assessing nasal protection by gently palpating the tip of the nose with the index finger. These were classified as good, moderate or poor protection. Also by palpation, the presence or absence of the "I-Beam" was assessed and any scarring of the columella or auricle was noted. The subjective degree of postoperative functional satisfaction regarding quality of breathing and smell perception were evaluated. Patients were also required to quantify their overall satisfaction (functional and cosmetically) with the surgery. Quantification was performed marking the answers in a visual analogue scale $(0-10$, zero being the most negative answer, and ten being the most positive one). Based on the position of the marking, answers were divided in classes according to quartiles: zero to $<2.5$ (very poor), 2.5 to $<5$ (poor); 5 to $<7.5$ (good); and $>7.5$ (very good).

\section{Statistical analysis}

Statistical analyses were performed with GraphPad Prism version 6.00 for Mac OS X, GraphPad Software, La Jolla, CA, USA (www.graphpad.com). Data are presented as means and standard deviation unless stated otherwise. The paired T test (checked by Kolmogorov-Smirnov test) was used to compare the mean scores between pre- and postoperative digital images. A value of $p<0.05$ was considered statistically significant, whereas values of $p<0.01$ were defined as highly significant.

\section{Results}

The "I-Beam" graft was performed in 72 patients undergoing septorhinoplasty over an eight year period. All patients had 


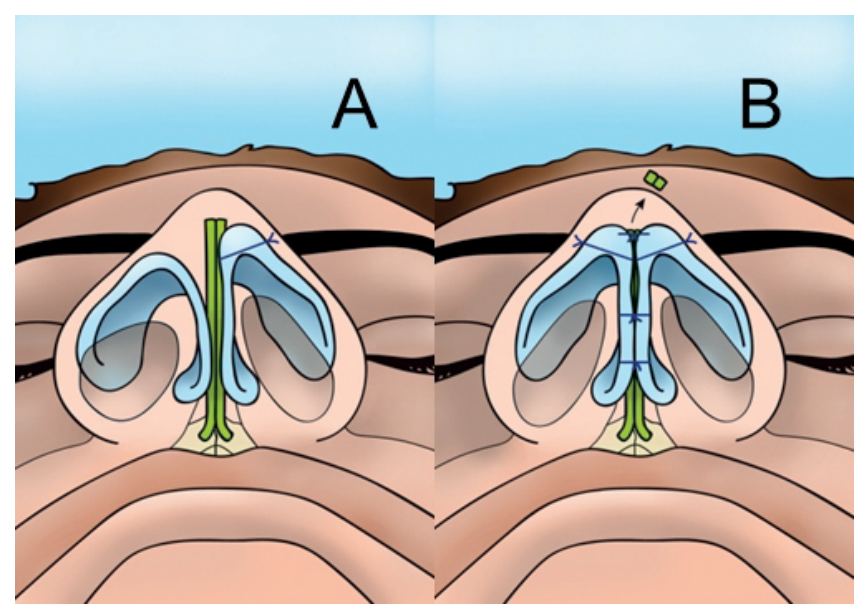

Figure 4. A) Transfixion sutures secure the "I-Beam" safely between the medial crura of the lower lateral cartilages. B) This can be combined with dome sutures.

a severe and significant loss of nasal tip protection, lack of remaining septal cartilage and the need of reconstruction of the caudal septum and the nasal tip support. Four patients with incomplete charts or photo-documentation were excluded from the study, two patients could not assist to the follow-up because of emigration. The biometrical analysis, the clinical examination, and the subjective analysis by questionnaire could be performed in 66 patients, being 18 women (27\%) and 48 men (73\%). The mean age was 35 years ( \pm 14 years). The mean follow-up was 89 months (min. 36, max. 143 months).

21 out of the total of 66 patients had a primary septorhinoplasty. A septoplasty alone was performed in 9 (14\%) patients. The main reason for a lack of caudal septum in these primary surgeries was due to a nasal trauma in 22 patients, a septal abscess in 6 patients, a cocaine nose in 1 patient and a relapsing polychondritis in 1 patient. The remaining 36 patients had a revision septorhinoplasty after one or several previous surgeries. The main reason for a lack of caudal septum in this group was due to multiple previous surgeries in 28 patients, a septal perforation in 5 patients, a cleft nose in 2 patients and a relapsing polychondritis in 1 patient. A submucous turbinoplasty in cases with inferior turbinate hypertrophy was performed in 27 (41\%) patients.

\section{Biometric analysis}

Pre- and postoperative analysis of digital images (frontal and lateral view) could be performed and the nose tip projection was measured.

To quantify changes in the projection of the nose during the follow-up, digital images were analysed taking the diameter of the iris as a constant. Of all analysed patients, the preoperative nose projection was improved significantly from $13.80 \mathrm{~mm}( \pm$ $2.6 \mathrm{~mm}$ ) to $15,24 \mathrm{~mm}$ ( $\pm 2.86 \mathrm{~mm}$ ) in the long term follow-up $(p<0.0001)$. In females, the preoperative nose projection of

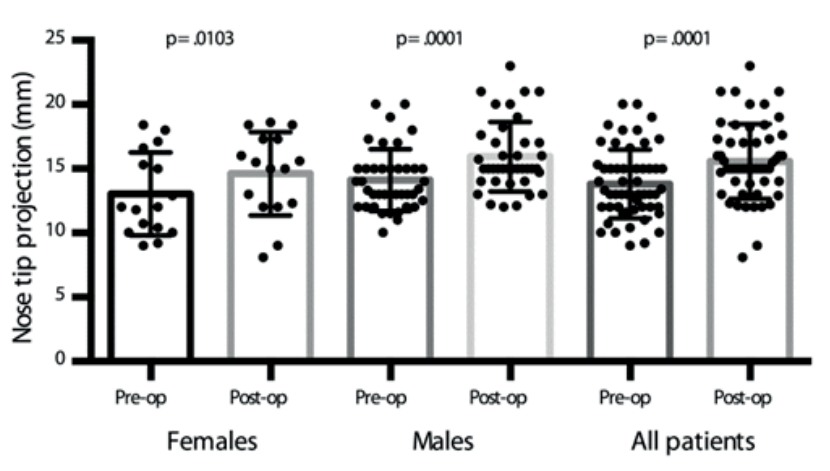

Figure 5. Boxplot showing preoperative and postoperative nasal tip projection.

$13.03 \mathrm{~mm}$ ( $\pm 3.13 \mathrm{~mm}$ ) improved to $14.6 \mathrm{~mm}( \pm 3.15 \mathrm{~mm})$ after surgery. In males, correction of the nose projection was also significant, from $14.48 \mathrm{~mm}( \pm 2.32 \mathrm{~mm}$ ) to $15.90 \mathrm{~mm}$ ( $\pm 2.66 \mathrm{~mm}$ ) postoperatively (Figure 5).

An example of septorhinoplasty using the "I-Beam" graft in a patient with a severe saddle nose deformation after trauma and previous surgeries is seen in Figure 6.

Subjective functional satisfaction and clinical examination Of the 66 patients examined, the overall surgical success was rated as very high by 52 patients (79\%), as high by 3 patients (5\%) and moderate by 8 patients (12\%). 3 patients (5\%) were not satisfied. Subjective individual analysis of the function of the nose after surgery was performed. Compared with preoperative breathing surgery improved the breathing quality in 57 (86\%) while in 9 patients it did not (14\%). Divided in subgroups, patients with additional submucous turbinoplasty did not differ in improving nasal breathing from patients without. Smell perception was subjectively evaluated and reported as very good in 33 patients (50\%), good in 22 (33\%), poor in $8(12 \%)$ and very poor in $3(4 \%)$. In 16 patients (24\%), the smell perception improved after surgery while in $50(76 \%)$ it remained unchanged. No subjective worsening in the smell perception was reported. In the clinical examination, the nose tip protection was good in 54 patients (82\%), moderate in $10(15 \%)$ and poor in 2 patients (3\%). By means of palpation, the "I-Beam" was found within the columella in all but one patient. This exception was a 56-year-old female on her 83th month follow-up suffering from relapsing polychondritis, which had again affected the anterior third of the nose. In the further 65 patients the "I-Beam" could be palpated within the columella. By the time of examination, two patients had had functional revision surgery at 4th and 6th postoperative year respectively to reposition a luxated "I-Beam" that had produced nasal obstruction.

\section{Complications}

No obvious scaring of the columella or in the nasal vestibule 


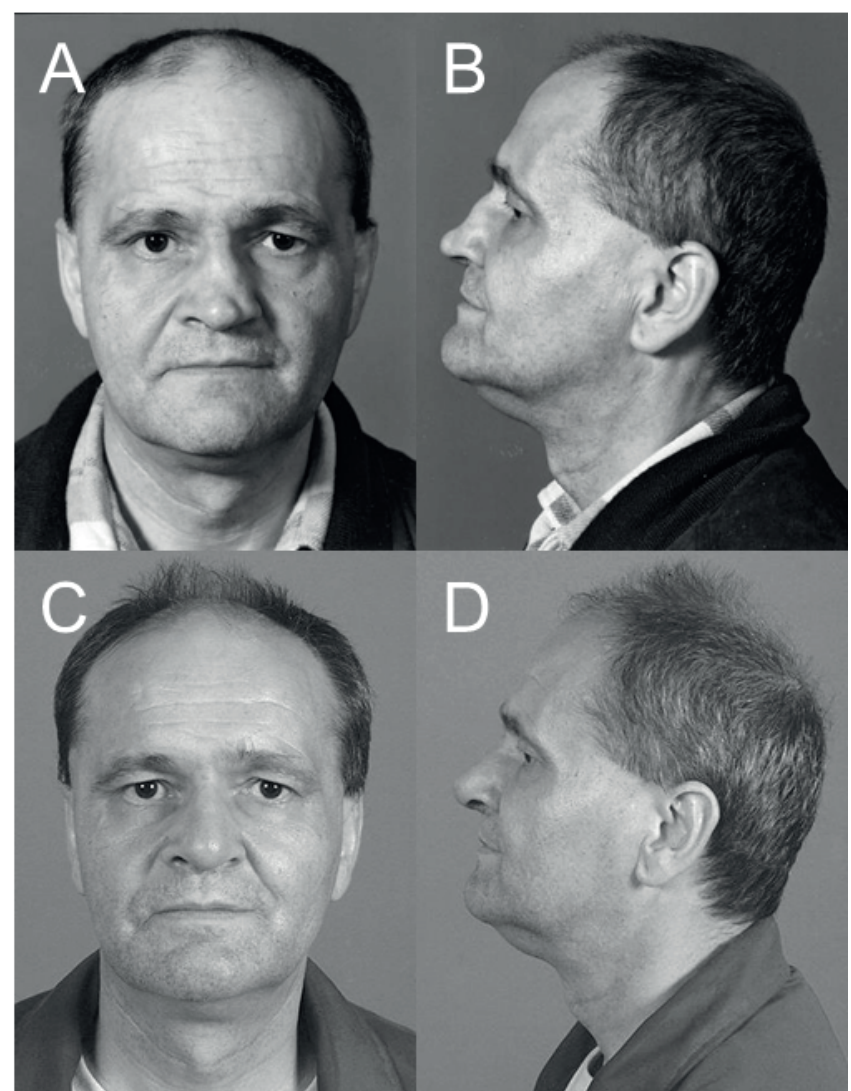

Figure 6. Reconstruction of a saddle nose after severe trauma. The "I-Beam" technique improves significantly nasal tip projection and columellar show: preoperative front $(A)$ and side view (B), postoperative front (C) and side view (D).

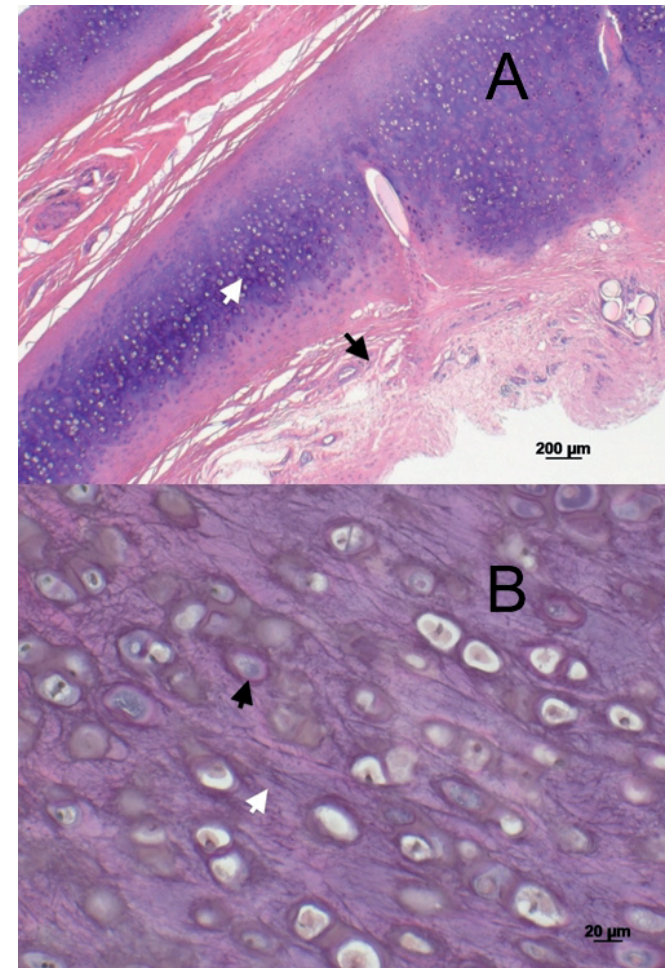

Figure 7. Biopsy of an "I-Beam" 10 years after primary surgery. A) Strong, vital cartilage (white arrow) is surrounded by soft tissue (black arrow) B) The histological examination shows the typical characteristics of the elastic conchal cartilage given by presence of abundant elastic fibers in the extracellular matrix (white arrow) between the chondrocytes (black arrow) when stained with H\&E. No signs of resorption, degeneration, calcification or hyaline transformation are observed. was seen in any of the 66 patients of this series. A retroauricular keloid that required revision surgery and a skin graft was seen in 1 patient. Revision surgery for repositioning the "I-Beam" was performed in two patients with excellent functional results as described. During revision rhinoplasty correcting a dislocated dorsal onlay graft we had the opportunity to take a biopsy of an "I-Beam" 10 years after primary surgery. Histological analysis showed the typical characteristics of the elastic conchal cartilage given by presence of abundant elastic fibres in the extracellular matrix between the chondrocytes in lacunae when stained with H\&E. No signs of resorption, degeneration, calcification or hyaline transformation were observed (Figure 7).

\section{Discussion}

Sufficient nasal tip support needs a stable foundation by a strong caudal septum (regio I). Multiple methods have been described to reconstruct this part of the septum using septal, auricular or rib cartilage as autologous grafts or even implants. The autologous conchal cartilage constitutes our primary choice for restoring nasal tip support due to the loss of the caudal septum in cases where septal cartilage is not available or of poor quality. This technique can be combined with all other types of rhinoplasties correcting deviated, crocked, hump or saddle noses. In our long-term study with a mean follow-up of more than seven years we could evaluate and confirm the long standing high functional, strong structural and good cosmetic results of the "I-Beam" graft with showing a long-lasting nasal tip protection and a highly significant improvement in nasal tip projection. All our patients received an autologous conchal cartilage graft in order to restore tip support. This "I-Beam", a structural intercrural columellar strut graft, is built by back-to-back sutured conchal cartilage and is placed in a circumferential soft tissue columellar pocket. The surrounding soft tissue not only makes the strut more secure but helps bolster the columella and support the columella incision. This may result in less infections and a better columellar show, when the columella is retracted. Furthermore, the perichondrium stays intact in the "I-Beam" technique and may give additional protection and structural strength ${ }^{(9)}$. In our series we had one loss of an "I-Beam" graft not due to an infection, but due to a relapsing polychondritis. In contrast to the technique described by Pirsig in $2004{ }^{(6)}$ the "I-Beam" is not sutured to one side of the remaining nasal septum like a septal 
extension graft, but stands alone in the columella giving more flexibility. The advantages of the "I-Beam" include very good support to the nasal tip and the lower lateral cartilages, filling any retraction of the NLA, enhancing columellar show and giving the sensation of natural elasticity when the nose is touched. This study provides objective measurements that allow a comparison of the pre - and postoperative nasal tip projection as previously described ${ }^{(5)}$. This demonstrates that the "I-Beam" graft significantly improved projection of the nose in all our patients. Although transdomal and interdomal sutures can impact projection in rhinoplasties with intact nasal tip support, this mechanism plays a very minor role in our series of difficult noses with total lack of tip support. The main mechanism for tip support in these cases is the structural strength of the "I-Beam" graft.

The long-term follow-up of our study ranging from 36 up to 143 months (mean 89 months) allowed us to evaluate alterations of the nose over several years and evaluate whether cartilage reabsorption occurred. Early animal studies showed that autologous rabbit auricular cartilage is resorbed slightly more than rib cartilage over twelve months. Interestingly, no statistically significant differences in resorption of cartilage occurred between the untouched, carved, and crushed cartilage ${ }^{(10)}$. In our series we had no signs of any cartilage resorption in clinical examination in all but one patient. We could furthermore study one "I-Beam" graft histologically 10 years after surgery that was sampled while relocating a displaced "I-Beam" graft. The cartilage showed histological signs of being viable whilst preserving its characteristic elastic architecture without any signs of resorption or hyaline transformation. In our evaluation only one of the patients showed signs of cartilage loss over the years. This case was a female patient with relapsing polychondritis. In the follow-up performed 83 months after surgery there was an evident loss of the cartilage in this patient, which included the "I-Beam". The limited literature on this subject ${ }^{(11)}$ indicates that in relapsing episodes of nasal chondritis immunosuppressive therapy may lower the rate of resorption.

The incidence of complications with the "I-Beam" technique was low. In two cases the "I-Beam" needed to be repositioned due to subluxation and in one patient a skin graft needed to be performed due to large retroauricular keloid.

Restoration of nasal tip support with the autologous back-toback sutured auricular cartilage "I-Beam" graft as a structural intercrural columellar strut graft in a circumferential soft tissue pocket constitutes an excellent and safe surgical method with high long-term functional and cosmetic improvement. It can be combined with all type of reduction or augmentation rhinoplasties.

\section{Acknowledgements}

Dr. Franziska Keller from the Institute of Pathology at the Luzerner Kantonsspital did the histological analysis of the cartilage biopsy. Professor Nick Jones, Nottingham, helped with revising the manuscript.

\section{Authorship contribution}

EN: study design, data collection, statistical analysis, composition of manuscript; DS: study design, composition and review of manuscript; HB: study design, composition and review of manuscript; CS: study supervision, surgeon, data collection, statistical analysis, composition of manuscript.

\section{Conflict of interest}

None.

\section{References}

1. Tardy ME, Thomas JR. Rhinoplasty. In Cummings C.W. Fredickson JM, Harker LA, et al., eds. Otolaryngology-Head \& Neck Surgery. 5 th edition. Philadelphia. Mosby, 2010; 508-544

2. Lekakis G, Dekimpe E, Steelant B, Hellings PW. Managing nasal valve compromise patients with nasal dilators: objective vs. subjective parameters. Rhinology 2016; 54: 348-354

3. Lovice DB, Mingrone MD, Toriumi DM. Grafts and implants in rhinoplasty and nasal reconstruction. Otolaryngol Clin North Am.1999;32(1):113-141.

4. McCollough E.G: Nasal Plastic Surgery, Saunders, 1994. ISBN-13: 9780721640679

5. Spoerri S, Simmen D, Briner HR, Jones N Objective assessment of tip projection and the nasolabial angle in rhinoplasty. Arch
Facial Plast Surg. 2004;6(5):295-298.

6. Pirsig W, Kern EB, Verse T. Reconstruction of anterior nasal septum: back-to-back autogenous ear cartilage graft. Laryngoscope 2004; 114(4):627-638.

7. Erdem T. Long-term effectiveness of projection control suture in rhinoplasty. Rhinology 2010; 48: 189-194

8. Crumley RL, Lanser M. Quantitative analysis of nasal tip projection. Laryngoscope 1998;98(2):202-208

9. Tekke NS, Alkan Z, Yigit O, Bekem A, Unal A, Sahin $\mathrm{F}$ et al. Importance of nasal septal cartilage perichondrium for septum strength mechanics: a cadaveric study. Rhinology 2014; 52: 167-171

10. Tjelmeland K, Stal S. Cartilage graft resorption: An animal model. Aesthetic Surgery Journal. 2000;20(6):471-476.

11. Sachse F, Stoll W. Nasal surgery in patients with systemic disorders. GMS Curr Top Otorhinolaryngol Head Neck Surg. 2010; 9:1-15.

Dr. med. Christoph Schlegel

Department of Otorhinolaryngology Head \& Neck Surgery Luzerner Kantonsspital 6000 Lucerne 16

Switzerland

Tel: +41-41-205 4993

Fax: +41-41-205-2151

E-mail: christoph.schlegel@luks.ch 